\title{
DEVELOPMENT OF CBR-BASED ROAD CONSTRUCTION PROJECT COST ESTIMATION SYSTEM
}

\author{
Tai Kyung Kang ${ }^{1}$, Wonyoung Park $^{1}$, and Yoo Sub Lee ${ }^{2 *}$ \\ ${ }^{1}$ Cost Engineering Research Division, Korea Institute of Construction Technology, Korea \\ ${ }^{2}$ International Cooperation \& Information Service Department, Korea Institute of Construction Technology, \\ Korea \\ *Corresponding author (yslee1@kict.re.kr)
}

\begin{abstract}
Compared with the method of predicting the detailed construction cost based on the information of the detailed quantities derived from the completed work drawings and specifications, the method of predicting the approximate estimate based on facility specification or approximate design information, etc. at the stage before the drawings and specifications are completed tends to rely greatly on the subjective judgment of the person in charge of construction cost prediction, which is low in its objectivity accordingly.

This study is aimed at exploring approximate estimate prediction model by types and stages of construction work, and then developing a WEB-based approximate estimation system thereon. This system is based on a scientific methodology such as CBR or regression model, etc. by means of facility specification and construction work characteristics in predicting the typical work type approximate quantities or approximate estimate, and is therefore expected to contribute to improving the accuracy in the prediction of approximate estimate and the advancement of such process. In addition, there would be an effect from the aspect of transparency of business as the user can easily access to the internet and receive the approximate estimate prediction result in an objective and consistent manner.
\end{abstract}

Key Words: Approximate Estimate, Approximate Quantities, Case Based Reasoning (CBR)

\section{Introduction}

\subsection{Background and Necessity of the Study}

In planning and carrying out the construction work, the construction cost is the element that may have important influence on many-sided decision makings. The feedback of the systematic construction cost information is necessary in assessing the economy based on the efficiency against investment at the early stage of the project when the business is planned or comparing the alternatives derived from the assessment of economy or advancing the design. In this connection, it is necessary to take note of the fact that the influence of the result of decision-making on the construction cost rather gets larger as it reaches the early stage of project such as planning and design, etc.

Nevertheless, in the case of public construction project in Korea, compared with the process of predicting the construction cost based on the detailed quantities information after the drawings and specifications are completed, the process of predicting the construction cost approximately at the early stage is too low in objectivity and transparency, which require an improvement. Such low accuracy of the construction cost predicted at the early stage inevitably comes from the difference in volume and level of the information that can be used in construction cost prediction, but its improvement is urgently needed from the aspect that it is vulnerable in terms of consistency as well as objectivity and transparency of approximate estimate prediction at the early stage.

The advancement in the process of predicting the construction cost at early stage is also an important task that is the first step to efficiently execute the finance of the government after all.

\subsection{Range and Purpose of the Study}


This study was aimed at developing an approximate estimate prediction system that is useful for coping with presuming the approximate budget at the planning stage for the facilities such as highway, stream, office building, apartment, etc. or predicting the approximate estimate for the purpose of design alternative comparison. The prediction of approximate budget was based on construction cost prediction model using facility specification and construction work characteristics while the approximate estimate prediction model to compare the design alternative based on the typical work type approximate quantities. The construction cost prediction model based on the entire work type detailed quantities was, however, excluded for the reason that the commercial software has been widely distributed to the relevant working process such as preparation of standard price, etc. (See Fig. 1)

Efforts were made to enhance the accuracy by adopting CBR or regression analysis, etc. in the case of approximate estimate prediction model applied to the system, and the access of the users was improved through the development of WEB-based system.

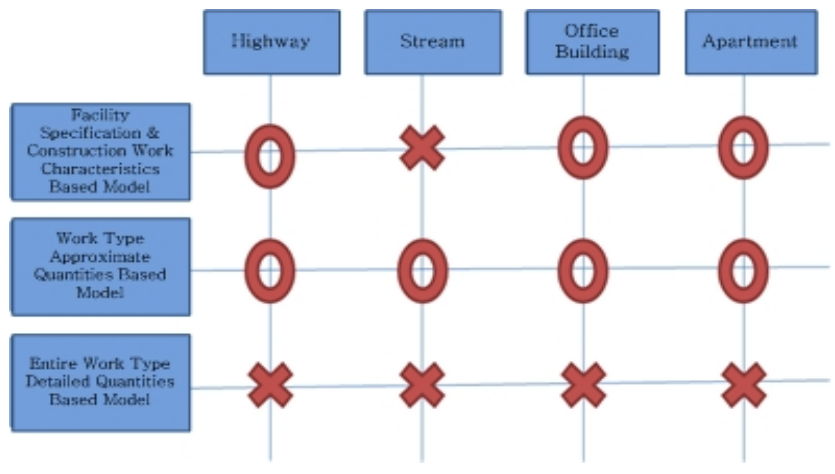

Fig. 1 Category supported by approximate estimate system

\section{Theoretical Ground of Approximate Estimating System}

2.1 Differentiation of Model in Accordance with the Level of Information Available at the Stage of Construction Work

The construction work is planned and executed over a long period of time throughout the stages of planning, design, construction, etc., and therefore there exists difference in the level of information that can be used for the prediction of construction cost by stages. Fig. 2 shows the general models used in the prediction of construction cost by stages, major users, and the types of construction cost information used in the construction cost prediction model.

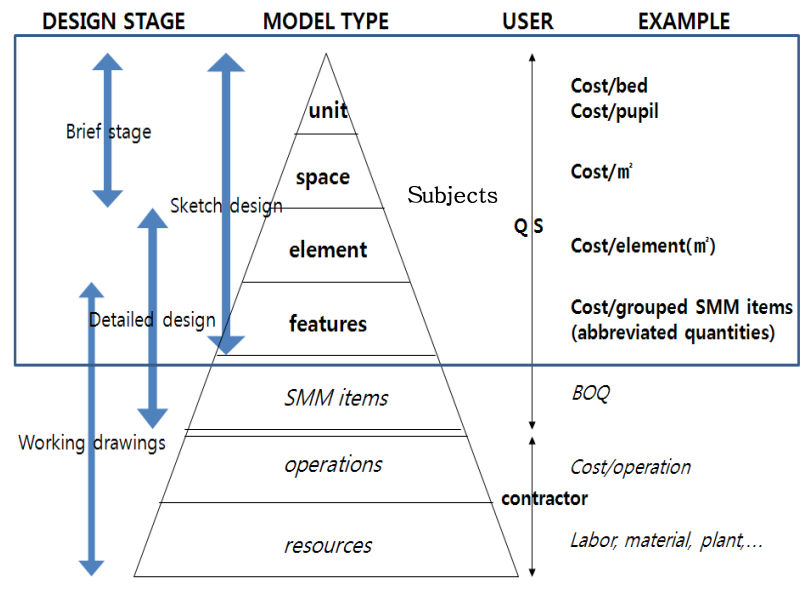

*SMM (Standard Method of Measurement)

** Cost Planning of Buildings $7^{\text {th }}$ Edition, pp. 113, [3]

Fig. 2 Construction work phase-wise cost prediction model

As shown in the Fig. 2 as above, this study is aimed at the early-stage models that are difficult to apply the detailed estimate prediction model based on the detailed quantities by work types and the unit prices constituting the cost breakdown. In the Figure, generally speaking, the approximate estimate prediction models based on unit, space, element, etc. are all the facility specification and construction work characteristics based model, both of which are similar to each other from a formal aspect. Therefore, the approximate estimate prediction model at the early stage can be broadly divided into facility specification or construction work characteristics based model and the approximate quantities based model of the typical work type.

\subsection{Case based Reasoning}

Generally speaking, the concept of CBR (Case Based Reasoning) characterized by its way of applying the previous cases accumulated to the solution of the problems in a new case and its verification is the ground that goes through the entire approximate estimate prediction model suggested in this study.

The case-based reasoning process adopted by approximate estimate prediction model follows the procedure of giving weighted value by characteristic variables, calculating 
similarity score of the previous case, and referring to the information of the estimate of the case with high similarity. Fig. 3 shows the procedure of predicting the approximate estimate of national highway taking advantage of CBR concept, provided that, however, there exists variables that are taken into account depending on the types of facility and the procedural difference. On the other hand, decision on weighted value becomes an important issue in the calculation of similarity score of the previous case. This study was intended to suggest weighted value by applying GA (Genetic Algorithm) methodology.

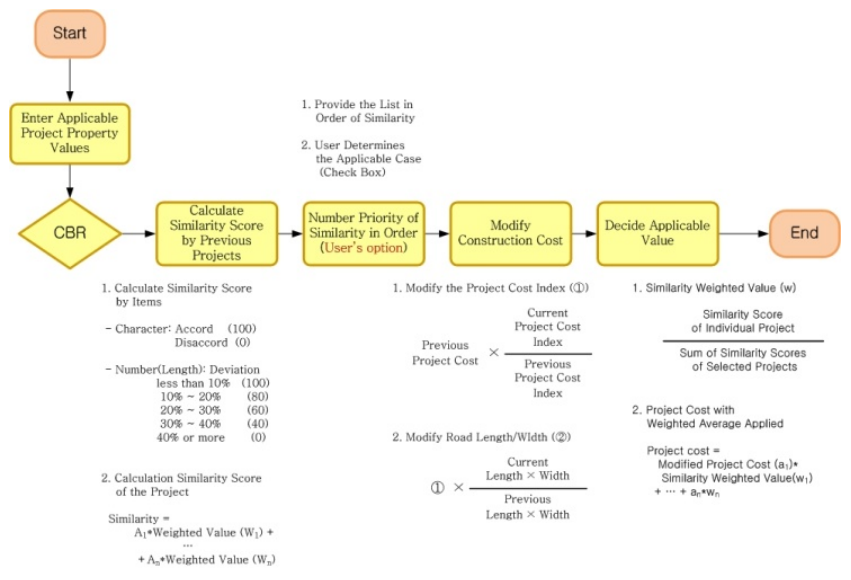

Fig. 3 Procedure of predicting the approximate estimate based on CBR

Nevertheless, the concrete CBR algorithm or weighted value estimation model was not mentioned in this study as they were not the key theme of this study.

\subsection{Pareto Principle}

"Pareto principle" the name of which is derived from Pareto - an Italian economist can be summarized into " $20 \%$ of vital few governs the remainder $-80 \%$ of the trivial many". Of course, the ratio could be either $20: 80$ or 10:90 depending on the fields, but the theory that minor governs majority is deemed valid from the aspect of construction estimate analysis too. That is, some expenses of typical types of work occupy the majority of the estimate.

From such aspect, approximate estimate prediction model based on typical work type approximate quantities can be the results from the application of Pareto principle concept to the cost estimation, in which, the construction cost is estimated for the major items in relatively a precise manner using the volume and the latest unit price at the time of estimation while for the minor items in a lump sum manner at a certain ratio.

In addition, Pareto principle was applied for the calculation of unit price by typical items, that is, for the minor items among a variety of items constituting the unit prices, it adopted the method of calculating their expenditure based on a certain ratio only. Fig. 4 shows the example of unit price calculation that was adopted in approximate construction cost estimating system where the unit price in the items of "re-bar preparation/assembly" is mainly composed of the labor cost spent for preparation and assembly. The material cost of bending wire was less than $1 \%$ of the total cost, but the survey/application of unit price was not compliant with the basic intent of approximate estimate prediction. Therefore, this study adopted the method of calculating the bending wire cost based on the ratio of bending wire to preparation/assembly labor cost in the approximate construction cost estimating system.

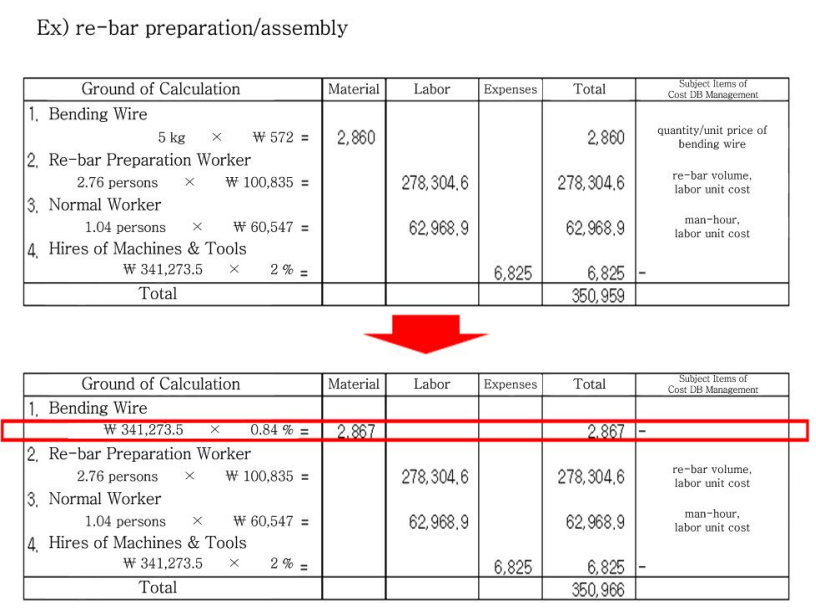

Fig. 4 Application of Pareto principle to the ground of estimation

\section{WEB-based Construction Cost Estimation System}

\subsection{Service Type and System Flow}

In developing approximate construction cost estimating system, this study was aimed at developing the WEB-based system so that the users can make use of it anywhere in an internet accessible environment (See Fig. 5). 
The flow of WEB-based approximate construction cost estimating system supports facility specification or construction work characteristics based model and the typical work type approximate quantities based model, and the flow of approximate construction cost estimating system for the national highway was shown in the Fig. 6.

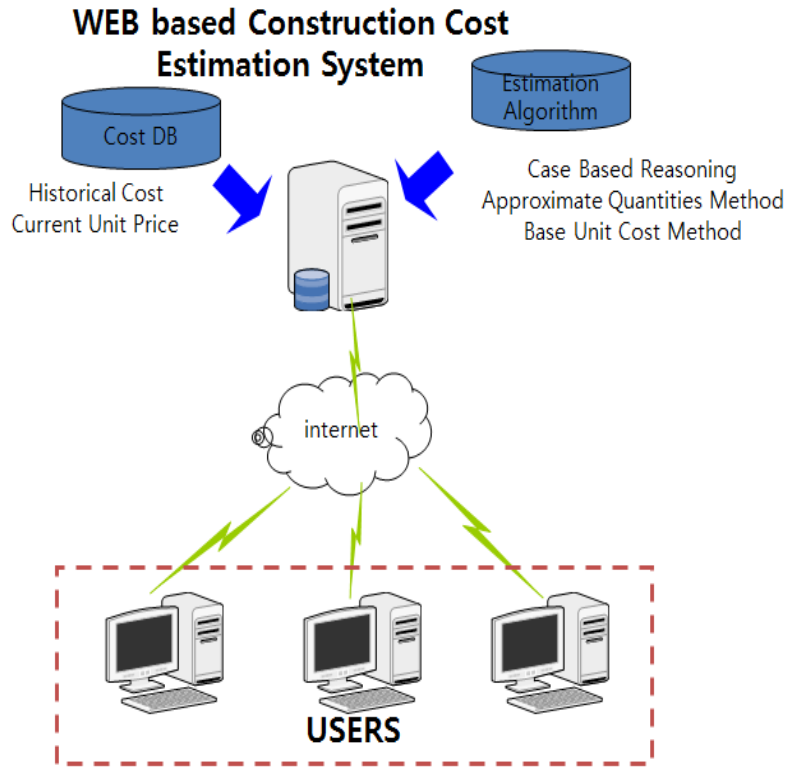

Fig. 5 Service pattern of the approximate construction cost estimating system

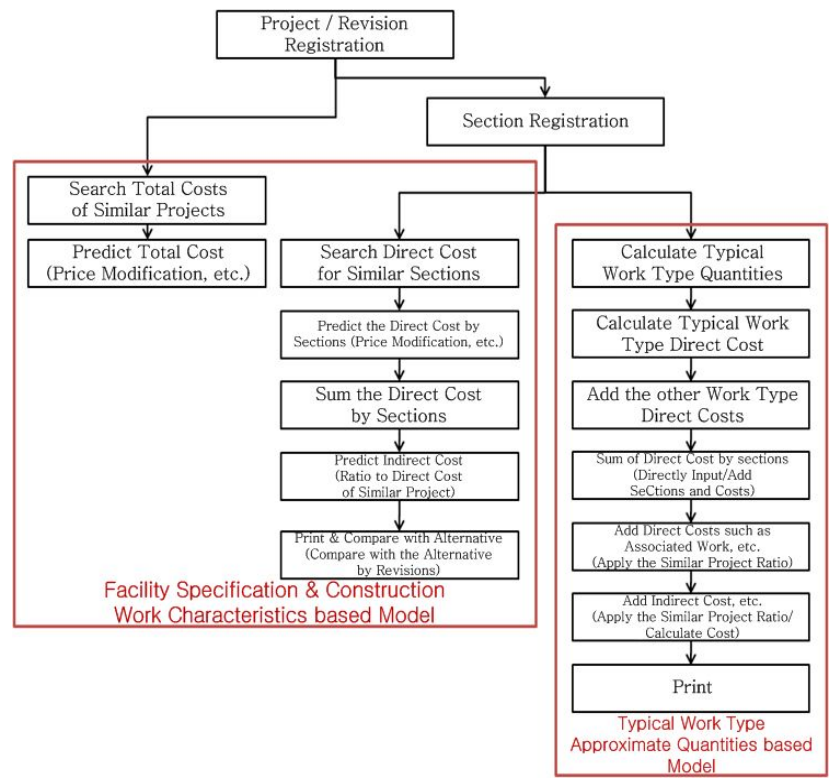

Fig. 6 Flow of approximate construction cost estimating system (National Highway)
Facility specification and construction work characteristics based approximate estimate prediction models are done in such a flow that searches and suggests the information of the similar project/zone construction cost from the system on the basis of the facility specification and construction work characteristics information entered by the user when it registered the project and the zones.

In the approximate estimate prediction model based on the typical work type approximate quantities, the user's role was similar, but it required entering the construction work characteristic information in more concrete and particular manner than the case of facility specification and construction work characteristics based model.

\subsection{User Interface}

\subsubsection{Facility Specification and Construction Work}

\section{Characteristics based Model}

As shown from the case of approximate construction cost estimating system in the national highway field, the facility specification and construction work characteristics based approximate estimate prediction model can be broadly divided into the function of predicting approximate estimate of the entire route including highway structures and the function of predicting the approximate estimate by sections constituting the route (civil engineering work section, bridge section, tunnel section, etc). For the purpose of this study to estimate the approximate budget cost at the early stage for the project planning, the interface of the system is very simple.

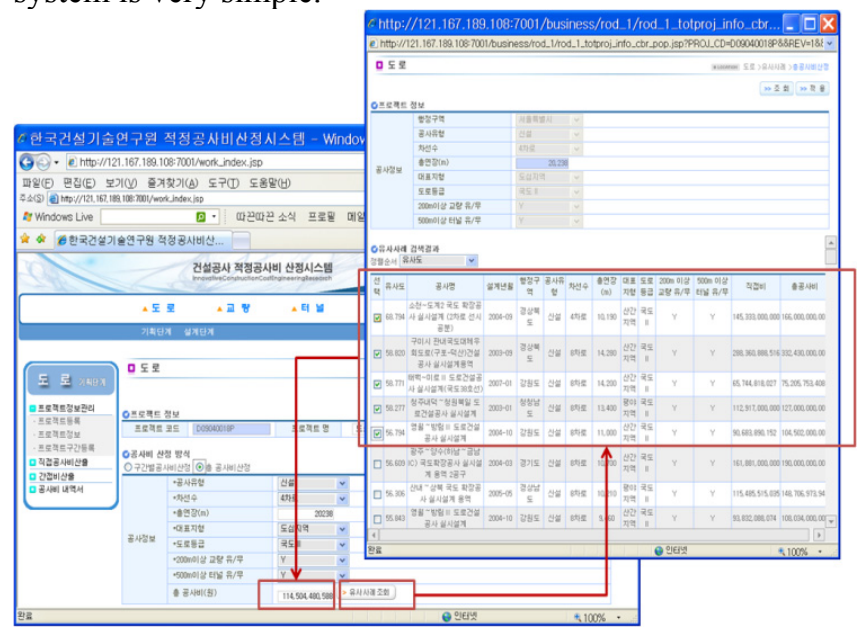

Fig. 7 Function of predicting the approximate estimate of the entire highway route 
Fig. 7 shows the screen of predicting the approximate estimate of the entire route of the national highway. When the user enters the variables such as number of lanes, total length, topographical characteristics, highway rating, existence of lengthy bridge and tunnel, etc. of the applicable construction work, the system calculates the score of similarity by the previous routes based on this, and then suggest approximate estimate of the applicable case based on the construction cost for the similar route.

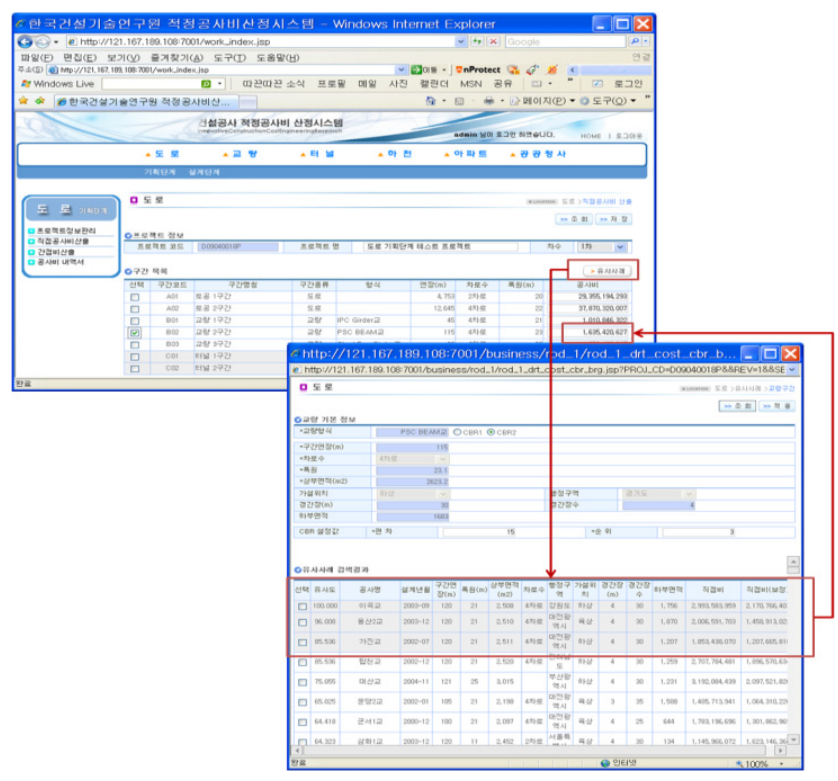

Fig. 8 Function of predicting the approximate estimate by highway sections

Fig. 8 shows a screen that predicts the approximate estimate by sections. The user is supposed to enter more detailed variables used in estimating the construction cost of the bridge section such as bridge type, location, span length, area of the lower part, etc. and then the system suggests the approximate estimate of the applicable bridge based on the construction cost of the previous similar bridge.

The variable needed to be input by the user vary depending on the facility, but the interfaces that the system suggests the approximate estimate of the applicable construction work based on the construction cost information of the previous similar cases are almost identical.

\subsubsection{Typical Work Type Approximate Quantities based Model}

Typical work type approximate quantities based model was developed for the purpose of construction cost comparison by design alternatives in the process of design being in progress, and therefore it has more complicate and precise interface than the facility specification and construction work characteristics based models have.

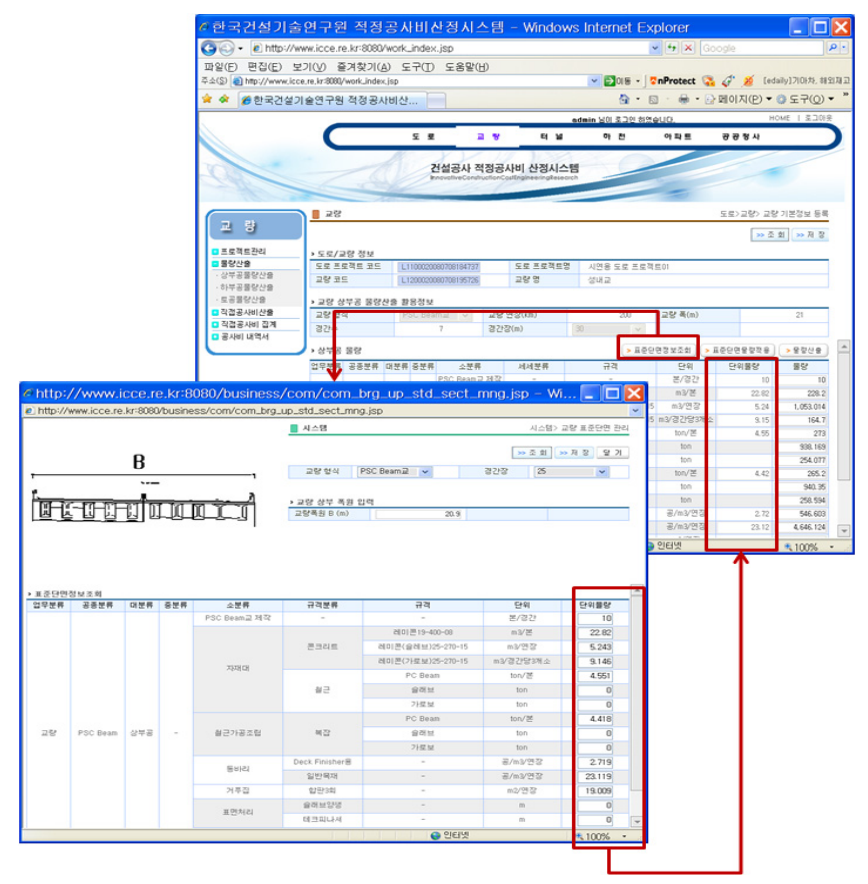

Fig. 9 Typical work type approximate quantities prediction algorithm (Bridge superstructure)

This study was intended for the system to estimate the typical work type approximate quantities based on the variables input by the user in advance. The approximate quantities by typical works are calculated by a variety of algorithms such as regression model or CBR, standard unit quantities table model, etc. depending on the characteristic of applicable typical works. Fig. 9 illustrates the function of predicting the approximate quantities by typical works taking advantage of standard unit quantities table and bridge specification information in connection with the standard drawing of the bridge superstructure.

After the approximate quantities by typical work types are calculated, the direct construction cost unit price to be applied to the quantities by typical work types can be estimated using the latest standard of estimate or historical cost data. (See Fig. 10) 
Here, the direct construction cost unit price by typical work types is divided into material cost, labor cost and expense so that the user can cope with the calculation of indirect construction cost, etc. later, which also provides the function of editing the variables influencing on the unit cost.

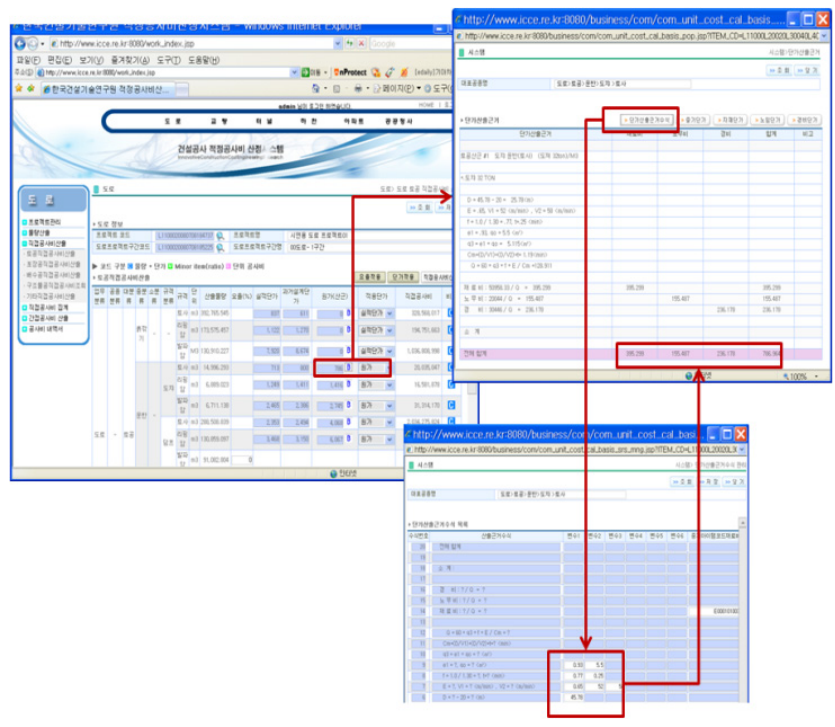

Fig. 10 Calculation of direct construction cost unit price by typical work types (Highway Civil Engineering Work)

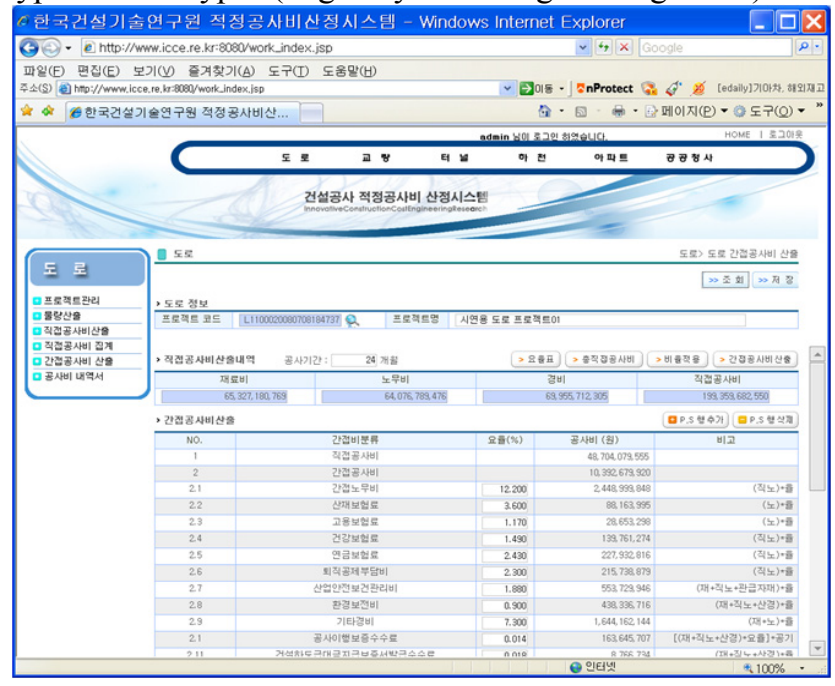

Fig. 11 Calculation of indirect construction cost (National Highway)

After the procedure of summing up with the typical work type-wise approximate quantities multiplied by the unit price, the direct construction cost of the work type with little importance will be added based on its ratio to the direct construction cost of the typical work type, and further the indirect construction cost is added by way of cost estimation on the basis of the summed direct construction cost. Fig. 11 shows a screen that calculates the indirect construction cost aimed at predicting the approximate estimate in the case of highway construction work.

\section{Conclusion}

As discovered from the foregoing, the approximate estimate prediction system suggested in this study was developed on the theoretical ground of CBR and Pareto principle. The variables of $\mathrm{CBR}$ or the breakdown of typical work type to be considered in detail, however, are differentiated in consideration of the types of construction work and the phase-wise characteristics.

The approximate estimate system of the construction work is expected to be distributed in a full scale from the second half of this year, through which, the basis for the advancement in the overall approximate estimate prediction will be probably established. First of all, this system, when used as a guideline on approximate estimate prediction, could enhance transparency, objectivity, consistency, etc. of approximate estimate prediction, and further it would result in great accessibility and availability to the user as it was developed in the form of WEB-based system.

\section{ACKNOWLEDGEMENT}

This research was supported by a grant (06 CIT A03) from Research Policy\&Infrastructure Development Program funded by Ministry of Construction \& Transportation of Korean government.

\section{REFERENCES}

[1] Allian A., "Cost Studies of Building", Pearson Prentice Hall, 1992

[2] Allan A. and Keith H., "Willis's Practice and Procedure for the Quantity Surveyor", Blackwell Publishing, 2007

[3] Douglas J.F., Peter S.B. and Jonathan D.F., "Cost Planning of Buildings”, Blackwell Science, 1999.

[4] Rodney D.S., Richard M.J., "Cost Estimator's Reference Manual", John Wiley \& Sons, 1995 